\title{
Nephrons are generated via a series of committed progenitors
}

Paola Romagnani, Laura Lasagni and Giuseppe Remuzzi

We thank Fujian Zhang and colleagues for their interesting comment (The Drosophila nephrocyte has a glomerular filtration system. Nat. Rev. Nephrol., 12 August 2014; doi:10.1038/nrneph.2012.290-c1) $)^{1}$ on our Review (Renal progenitors: an evolutionary conserved strategy for kidney regeneration. Nat. Rev. Nephrol. 9, 137-146; 2013). ${ }^{2}$ Several papers have highlighted similarities between the podocyte and the Drosophila nephrocyte; however, podocytes are an integral part of the nephron, whereas nephrocytes are spatially separated from renal (Malpighian) tubules. ${ }^{3,4}$ Although analogies between the Drosophila nephrocyte and the mammalian podocyte had been reported when we wrote our Review, the relationship between nephrocytes and Malpighian tubules had not been fully established. ${ }^{5}$ As highlighted in their correspondence, ${ }^{1}$ two studies published by Zhang et al. in 2013 strongly suggest that the nephrocyte is an integral constituent of the Drosophila kidney system and is functionally and molecularly related to mammalian podocytes and proximal tubular cells. ${ }^{6,7}$

The observation that Drosophila nephrocytes show phenotypic features of both mammalian podocytes and proximal tubular cells is particularly intriguing. In the human adult kidney, a subset of renal progenitors is bipotent and displays the potential to differentiate into podocytes ${ }^{8}$ and proximal tubular cells. ${ }^{8-10}$ The bipotent progenitor localizes at the urinary pole of the Bowman capsule and is characterized by the expression of the renal progenitor markers CD133 and CD24 in the absence of expression of lineage markers. Starting from this bipotent progenitor (which can be observed during kidney development from the vesicle stage onwards) all mature nephron epithelial cells emerge through a hierarchical series of lineage decisions via various committed progenitor cells. ${ }^{11}$ The intermediate cellular states are the tubular progenitors and the podocyte progenitor. The tubular progenitors, characterized by expression of CD133 and CD24 as well as low levels of tubular progenitor markers, can proliferate and differentiate into proximal tubular cells, cells of the loop of Henle, distal tubular cells and cells of the connecting segment, through a series of cell-typecommitted progenitors. ${ }^{9-10}$ In adult human kidneys these committed progenitors are localized as scattered cells along the proximal tubule (particularly the S3 segment), distal tubule and connecting segment. ${ }^{10}$ The podocyte progenitor localizes along the Bowman capsule and is characterized by coexpression of CD133, CD24 and low levels of podocyte markers. ${ }^{8}$ Podocyte progenitors can proliferate but can differentiate only into functional podocytes. ${ }^{8}$

Consistent with human data, lineage tracing experiments performed in our laboratory using the Confetti reporter system under the control of a progenitor-specific promoter, have confirmed that murine podocytes and tubular cells share a common progenitor during kidney development that generates precursors with more restricted potential, such as podocyte-committed or tubular-committed progenitors, and then generates a series of committed progenitors. ${ }^{12,13}$ Rinkevich et al. also recently proposed the existence within the nephron of a series of lineage-restricted progenitor cells that drive murine kidney development, maintenance and regeneration. ${ }^{14}$ In the zebrafish, recent data suggest that a common progenitor can generate podocytes and tubular cells through a graded series of committed progenitors. ${ }^{15,16}$ Interestingly, even the signalling pathways that control the proliferation and differentiation of renal progenitors toward the podocyte and proximal tubular lineage (which involve OSR $1,{ }^{15,17}$ Pax- $2,{ }^{16,18} \mathrm{WT} 1,{ }^{16} \mathrm{Wnt} / \beta$-catenin, ${ }^{14}$ Notch ${ }^{16,19}$ and retinoic acid ${ }^{16,20,21}$ ) seem to be highly conserved between zebrafish, mice and humans, further underlining the critical role of renal progenitors in kidney development and regeneration following injury.

Taken together, these observations suggest that the renal properties of filtration and protein reabsorption are closely related and probably evolved together in very simple organisms. However, when the structural complexity and requirements of organisms increased, these essential kidney functions required distinct cell types. Highly specialized cells generated from a common renal progenitor enabled the progressive separation of the functions of filtration and protein reabsorption through the generation of a graded series of committed progenitors that could eventually differentiate into podocytes and various subsets of tubular cells as observed in the mammalian kidney.

Pediatric Nephrology Unit, Meyer Children's Hospital, University of Florence, Viale Pieraccini 24, 50139 Florence, Italy (P.R.). Excellence Centre for Research, Transfer and High Education for the Development of De Novo Therapies (DENOTHE), University of Florence, Viale Morgagni 85, 50134 Florence, Italy (L.L.). Mario Negri Institute for Pharmacological Research, Via Stezzano 87, 24126 Bergamo, Italy (G.R.).

Correspondence to: P.R.

\section{paola.romagnani@unifi.it}

Acknowledgements

The authors' work is supported by the European Community under the European Community's Seventh Framework Programme (FP7/2012-2016), grant number 305436 .

Competing interests

The authors declare no competing interests.

1. Zhang, F. et al. The Drosophila nephrocyte has a glomerular filtration system. Nat. Rev. Nephrol. http://dx.doi.org/10.1038/nrneph.2012.290c1 (2014).

2. Romagnani, P., Lasagni, L. \& Remuzzi, G. Renal progenitors: an evolutionary conserved strategy for kidney regeneration. Nat. Rev. Nephrol. 9, 137-146 (2013).

3. Denholm, B. Shaping up for action: the path to physiological maturation in the renal tubules of Drosophila Organogenesis 9, 40-54 (2013).

4. Na, J. \& Cagan, R. The Drosophila nephrocyte: back on stage. J. Am. Soc. Nephrol. 24, 161-163 (2013). 


\section{CORRESPONDENCE}

5. Weavers, $\mathrm{H}$. et al. The insect nephrocyte is a podocyte-like cell with a filtration slit diaphragm. Nature 457, 322-326 (2009).

6. Zhang, F., Zhao, Y., Chao, Y., Muir, K. \& Han, Z. Cubilin and amnionless mediate protein reabsorption in Drosophila nephrocytes. J. Am. Soc. Nephrol. 24, 209-216 (2013).

7. Zhang F., Zhao, Y. \& Han, Z. An in vivo functional analysis system for renal gene discovery in Drosophila pericardial nephrocytes. J. Am. Soc. Nephrol. 24, 191-197 (2013).

8. Ronconi, E. et al. Regeneration of glomerular podocytes by human renal progenitors. J. Am Soc. Nephrol. 20, 322-332 (2009).

9. Lindgren, D. et al. Isolation and characterization of progenitor-like cells from human renal proximal tubules. Am. J. Pathol. 178, 828-837 (2011).

10. Angelotti, M. L. et al. Characterization of renal progenitors committed toward tubular lineage and their regenerative potential in renal tubular injury. Stem Cells 30, 1714-1725 (2012).
11. Lazzeri, E. et al. Regenerative potential of embryonic renal multipotent progenitors in acute renal failure. J. Am. Soc. Nephrol. 18, 3128-3138 (2007).

12. Romagnani, P. Stem cells and renal cell regeneration in acute kidney injury. Presented at the $51^{\text {st }}$ ERA-EDTA Congress (2014).

13. Romagnani, P. Podocyte regeneration: how it occurs and how to enhance it. Presented at the $10^{\text {th }}$ International Podocyte Conference (2014).

14. Rinkevich, Y. et al. In vivo clonal analysis reveals lineage-restricted progenitor characteristics in mammalian kidney development, maintenance, and regeneration. Cell Rep. 7, 1270-1283 (2014).

15. Diep, C. Q. et al. Identification of adult nephron progenitors capable of kidney regeneration in zebrafish. Nature 470, 95-100 (2011).

16. Kroeger, P. T. Jr \& Wingert, R. A. Using zebrafish to study podocyte genesis during kidney development and regeneration. Genesis http://dx.doi.org/10.1002/dvg.22798.
17. Zhang, Z. et al. A variant OSR1 allele which disturbs OSR1 mRNA expression in renal progenitor cells is associated with reduction of newborn kidney size and function. Hum. Mol. Genet. 20, 4167-4174 (2011)

18. Sallustio, F. et al. miR-1915 and miR-1225-5p regulate the expression of CD133, PAX2 and TLR2 in adult renal progenitor cells. PLOS ONE 8 , e68296 (2013).

19. Lasagni, L. et al. Notch activation differentially regulates renal progenitors proliferation and differentiation toward the podocyte lineage in glomerular disorders. Stem Cells 28, 1674-1685 (2010).

20. Li, Y., Cheng, C. N., Verdun, V. \& Wingert, R. A. Zebrafish nephrogenesis is regulated by interactions between retinoic acid, mecom, and Notch signaling. Dev. Biol. 386, 111-122 (2014).

21. Peired, A. et al. P. Proteinuria impairs podocyte regeneration by sequestering retinoic acid. J. Am. Soc. Nephrol. 24, 1756-1768 (2013). 